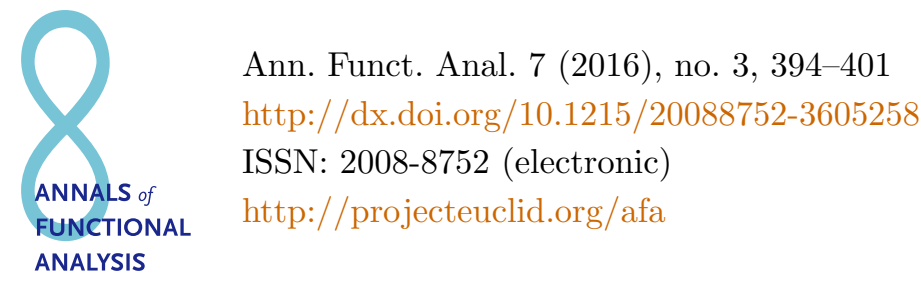

\title{
SOME TRACE MONOTONICITY PROPERTIES AND APPLICATIONS
}

\author{
JEAN-MICHEL COMBES ${ }^{1}$ and PETER D. HISLOP ${ }^{2 *}$ \\ Communicated by I. M. Spitkovsky
}

\begin{abstract}
We present some results on the monotonicity of some traces involving functions of self-adjoint operators with respect to the natural ordering of their associated quadratic forms. The relation between these results and Löwner's Theorem is discussed. We also apply these results to complete a proof of the Wegner estimate for continuum models of random Schrödinger operators as given in a 1994 paper by Combes and Hislop.
\end{abstract}

\section{Statement of the Problem And Result}

We consider two lower-semibounded self-adjoint operators $A$ and $B$ associated with closed symmetric, lower-semibounded quadratic forms $q_{A}$ and $q_{B}$ with form domains $Q(A)$ and $Q(B)$, respectively. We suppose that $q_{A} \leq q_{B}$. This inequality means that $Q(B) \subset Q(A)$ and that, for all $\varphi \in Q(B)$, we have $q_{A}(\varphi) \leq q_{B}(\varphi)$. Under these conditions, Kato proved in [8, Theorem 2.21, chapter VI] the following relationship between the resolvents of the two operators $A$ and $B$. For all $a>-\inf \sigma(A)$, we have

$$
(B+a)^{-1} \leq(A+a)^{-1} .
$$

This resolvent inequality may be used to derive several interesting relations between the traces of functions of $A$ and $B$ under some additional assumptions. We will prove that if $f \geq 0$ and $g$ is a member of a class of functions $\mathcal{L}$ described

Copyright 2016 by the Tusi Mathematical Research Group.

Received Aug. 3, 2015; Accepted Dec. 1, 2015.

${ }^{*}$ Corresponding author.

2010 Mathematics Subject Classification. Primary 47B10; Secondary 47A60, 47B80.

Keywords. operator trace inequalities, operator monotone functions, Loewner's Theorem, random Schrodinger operators, Wegner estimate. 


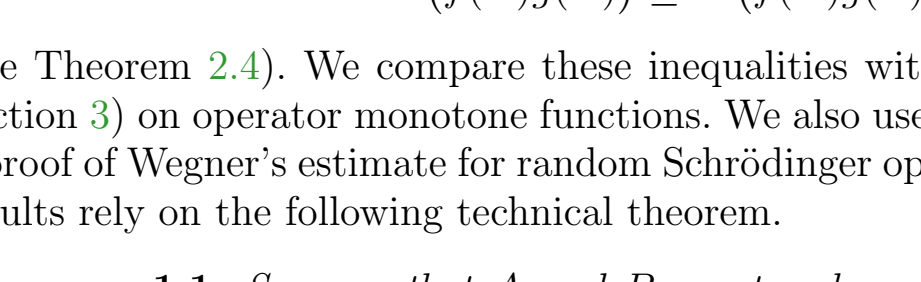

\title{
SOME TRACE MONOTONICITY PROPERTIES AND APPLICATIONS
}

\author{
JEAN-MICHEL COMBES ${ }^{1}$ and PETER D. HISLOP ${ }^{2 *}$ \\ Communicated by I. M. Spitkovsky
}

\begin{abstract}
We present some results on the monotonicity of some traces involving functions of self-adjoint operators with respect to the natural ordering of their associated quadratic forms. The relation between these results and Löwner's Theorem is discussed. We also apply these results to complete a proof of the Wegner estimate for continuum models of random Schrödinger operators as given in a 1994 paper by Combes and Hislop.
\end{abstract}

\section{Statement of the Problem And Result}

We consider two lower-semibounded self-adjoint operators $A$ and $B$ associated with closed symmetric, lower-semibounded quadratic forms $q_{A}$ and $q_{B}$ with form domains $Q(A)$ and $Q(B)$, respectively. We suppose that $q_{A} \leq q_{B}$. This inequality means that $Q(B) \subset Q(A)$ and that, for all $\varphi \in Q(B)$, we have $q_{A}(\varphi) \leq q_{B}(\varphi)$. Under these conditions, Kato proved in [8, Theorem 2.21, chapter VI] the following relationship between the resolvents of the two operators $A$ and $B$. For all $a>-\inf \sigma(A)$, we have

$$
(B+a)^{-1} \leq(A+a)^{-1} .
$$

This resolvent inequality may be used to derive several interesting relations between the traces of functions of $A$ and $B$ under some additional assumptions. We will prove that if $f \geq 0$ and $g$ is a member of a class of functions $\mathcal{L}$ described

Copyright 2016 by the Tusi Mathematical Research Group.

Received Aug. 3, 2015; Accepted Dec. 1, 2015.

${ }^{*}$ Corresponding author.

2010 Mathematics Subject Classification. Primary 47B10; Secondary 47A60, 47B80.

Keywords. operator trace inequalities, operator monotone functions, Loewner's Theorem, random Schrodinger operators, Wegner estimate. 


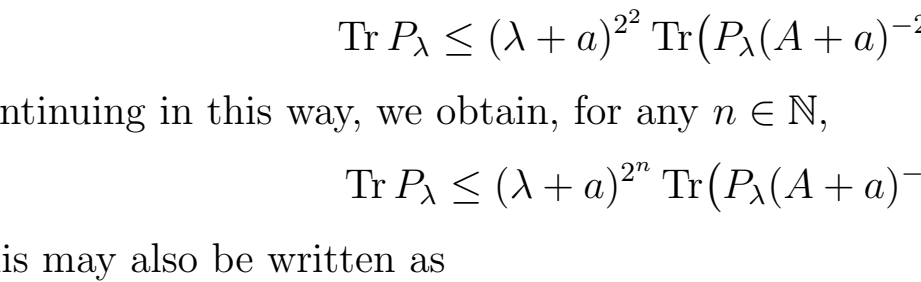

\title{
SOME TRACE MONOTONICITY PROPERTIES AND APPLICATIONS
}

\author{
JEAN-MICHEL COMBES ${ }^{1}$ and PETER D. HISLOP ${ }^{2 *}$ \\ Communicated by I. M. Spitkovsky
}

\begin{abstract}
We present some results on the monotonicity of some traces involving functions of self-adjoint operators with respect to the natural ordering of their associated quadratic forms. The relation between these results and Löwner's Theorem is discussed. We also apply these results to complete a proof of the Wegner estimate for continuum models of random Schrödinger operators as given in a 1994 paper by Combes and Hislop.
\end{abstract}

\section{Statement of the Problem And Result}

We consider two lower-semibounded self-adjoint operators $A$ and $B$ associated with closed symmetric, lower-semibounded quadratic forms $q_{A}$ and $q_{B}$ with form domains $Q(A)$ and $Q(B)$, respectively. We suppose that $q_{A} \leq q_{B}$. This inequality means that $Q(B) \subset Q(A)$ and that, for all $\varphi \in Q(B)$, we have $q_{A}(\varphi) \leq q_{B}(\varphi)$. Under these conditions, Kato proved in [8, Theorem 2.21, chapter VI] the following relationship between the resolvents of the two operators $A$ and $B$. For all $a>-\inf \sigma(A)$, we have

$$
(B+a)^{-1} \leq(A+a)^{-1} .
$$

This resolvent inequality may be used to derive several interesting relations between the traces of functions of $A$ and $B$ under some additional assumptions. We will prove that if $f \geq 0$ and $g$ is a member of a class of functions $\mathcal{L}$ described

Copyright 2016 by the Tusi Mathematical Research Group.

Received Aug. 3, 2015; Accepted Dec. 1, 2015.

${ }^{*}$ Corresponding author.

2010 Mathematics Subject Classification. Primary 47B10; Secondary 47A60, 47B80.

Keywords. operator trace inequalities, operator monotone functions, Loewner's Theorem, random Schrodinger operators, Wegner estimate. 


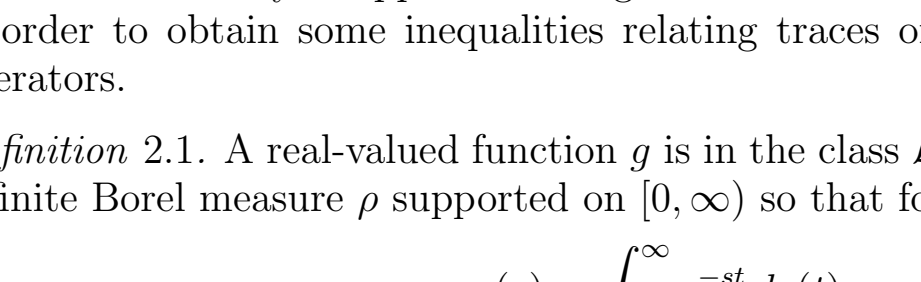

\title{
SOME TRACE MONOTONICITY PROPERTIES AND APPLICATIONS
}

\author{
JEAN-MICHEL COMBES ${ }^{1}$ and PETER D. HISLOP ${ }^{2 *}$ \\ Communicated by I. M. Spitkovsky
}

\begin{abstract}
We present some results on the monotonicity of some traces involving functions of self-adjoint operators with respect to the natural ordering of their associated quadratic forms. The relation between these results and Löwner's Theorem is discussed. We also apply these results to complete a proof of the Wegner estimate for continuum models of random Schrödinger operators as given in a 1994 paper by Combes and Hislop.
\end{abstract}

\section{Statement of the Problem And Result}

We consider two lower-semibounded self-adjoint operators $A$ and $B$ associated with closed symmetric, lower-semibounded quadratic forms $q_{A}$ and $q_{B}$ with form domains $Q(A)$ and $Q(B)$, respectively. We suppose that $q_{A} \leq q_{B}$. This inequality means that $Q(B) \subset Q(A)$ and that, for all $\varphi \in Q(B)$, we have $q_{A}(\varphi) \leq q_{B}(\varphi)$. Under these conditions, Kato proved in [8, Theorem 2.21, chapter VI] the following relationship between the resolvents of the two operators $A$ and $B$. For all $a>-\inf \sigma(A)$, we have

$$
(B+a)^{-1} \leq(A+a)^{-1} .
$$

This resolvent inequality may be used to derive several interesting relations between the traces of functions of $A$ and $B$ under some additional assumptions. We will prove that if $f \geq 0$ and $g$ is a member of a class of functions $\mathcal{L}$ described

Copyright 2016 by the Tusi Mathematical Research Group.

Received Aug. 3, 2015; Accepted Dec. 1, 2015.

${ }^{*}$ Corresponding author.

2010 Mathematics Subject Classification. Primary 47B10; Secondary 47A60, 47B80.

Keywords. operator trace inequalities, operator monotone functions, Loewner's Theorem, random Schrodinger operators, Wegner estimate. 


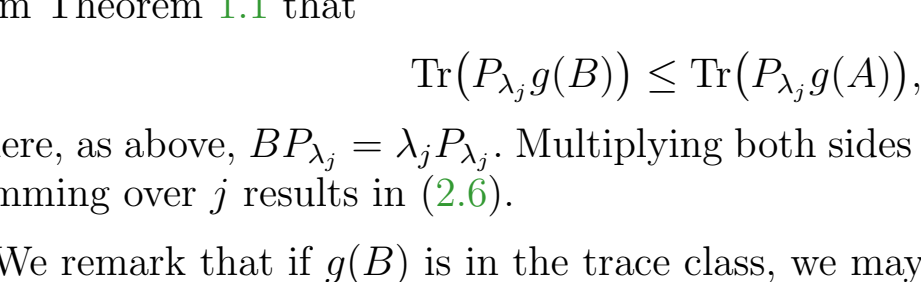

\title{
SOME TRACE MONOTONICITY PROPERTIES AND APPLICATIONS
}

\author{
JEAN-MICHEL COMBES ${ }^{1}$ and PETER D. HISLOP ${ }^{2 *}$ \\ Communicated by I. M. Spitkovsky
}

\begin{abstract}
We present some results on the monotonicity of some traces involving functions of self-adjoint operators with respect to the natural ordering of their associated quadratic forms. The relation between these results and Löwner's Theorem is discussed. We also apply these results to complete a proof of the Wegner estimate for continuum models of random Schrödinger operators as given in a 1994 paper by Combes and Hislop.
\end{abstract}

\section{Statement of the Problem And Result}

We consider two lower-semibounded self-adjoint operators $A$ and $B$ associated with closed symmetric, lower-semibounded quadratic forms $q_{A}$ and $q_{B}$ with form domains $Q(A)$ and $Q(B)$, respectively. We suppose that $q_{A} \leq q_{B}$. This inequality means that $Q(B) \subset Q(A)$ and that, for all $\varphi \in Q(B)$, we have $q_{A}(\varphi) \leq q_{B}(\varphi)$. Under these conditions, Kato proved in [8, Theorem 2.21, chapter VI] the following relationship between the resolvents of the two operators $A$ and $B$. For all $a>-\inf \sigma(A)$, we have

$$
(B+a)^{-1} \leq(A+a)^{-1} .
$$

This resolvent inequality may be used to derive several interesting relations between the traces of functions of $A$ and $B$ under some additional assumptions. We will prove that if $f \geq 0$ and $g$ is a member of a class of functions $\mathcal{L}$ described

Copyright 2016 by the Tusi Mathematical Research Group.

Received Aug. 3, 2015; Accepted Dec. 1, 2015.

${ }^{*}$ Corresponding author.

2010 Mathematics Subject Classification. Primary 47B10; Secondary 47A60, 47B80.

Keywords. operator trace inequalities, operator monotone functions, Loewner's Theorem, random Schrodinger operators, Wegner estimate. 


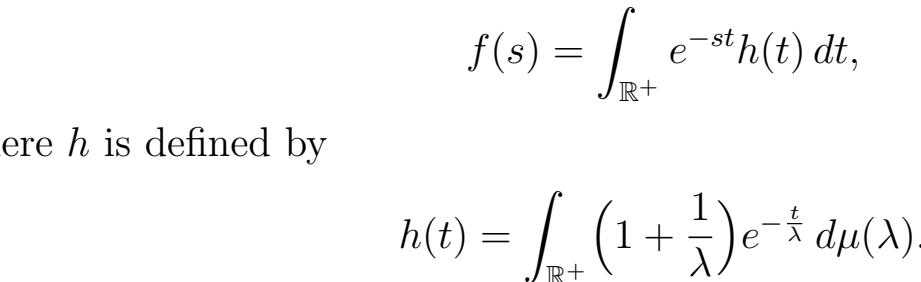

\title{
SOME TRACE MONOTONICITY PROPERTIES AND APPLICATIONS
}

\author{
JEAN-MICHEL COMBES ${ }^{1}$ and PETER D. HISLOP ${ }^{2 *}$ \\ Communicated by I. M. Spitkovsky
}

\begin{abstract}
We present some results on the monotonicity of some traces involving functions of self-adjoint operators with respect to the natural ordering of their associated quadratic forms. The relation between these results and Löwner's Theorem is discussed. We also apply these results to complete a proof of the Wegner estimate for continuum models of random Schrödinger operators as given in a 1994 paper by Combes and Hislop.
\end{abstract}

\section{Statement of the Problem And Result}

We consider two lower-semibounded self-adjoint operators $A$ and $B$ associated with closed symmetric, lower-semibounded quadratic forms $q_{A}$ and $q_{B}$ with form domains $Q(A)$ and $Q(B)$, respectively. We suppose that $q_{A} \leq q_{B}$. This inequality means that $Q(B) \subset Q(A)$ and that, for all $\varphi \in Q(B)$, we have $q_{A}(\varphi) \leq q_{B}(\varphi)$. Under these conditions, Kato proved in [8, Theorem 2.21, chapter VI] the following relationship between the resolvents of the two operators $A$ and $B$. For all $a>-\inf \sigma(A)$, we have

$$
(B+a)^{-1} \leq(A+a)^{-1} .
$$

This resolvent inequality may be used to derive several interesting relations between the traces of functions of $A$ and $B$ under some additional assumptions. We will prove that if $f \geq 0$ and $g$ is a member of a class of functions $\mathcal{L}$ described

Copyright 2016 by the Tusi Mathematical Research Group.

Received Aug. 3, 2015; Accepted Dec. 1, 2015.

${ }^{*}$ Corresponding author.

2010 Mathematics Subject Classification. Primary 47B10; Secondary 47A60, 47B80.

Keywords. operator trace inequalities, operator monotone functions, Loewner's Theorem, random Schrodinger operators, Wegner estimate. 


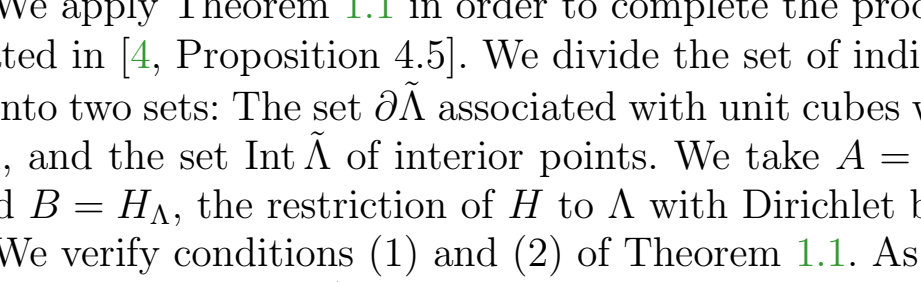

\title{
SOME TRACE MONOTONICITY PROPERTIES AND APPLICATIONS
}

\author{
JEAN-MICHEL COMBES ${ }^{1}$ and PETER D. HISLOP ${ }^{2 *}$ \\ Communicated by I. M. Spitkovsky
}

\begin{abstract}
We present some results on the monotonicity of some traces involving functions of self-adjoint operators with respect to the natural ordering of their associated quadratic forms. The relation between these results and Löwner's Theorem is discussed. We also apply these results to complete a proof of the Wegner estimate for continuum models of random Schrödinger operators as given in a 1994 paper by Combes and Hislop.
\end{abstract}

\section{Statement of the Problem And Result}

We consider two lower-semibounded self-adjoint operators $A$ and $B$ associated with closed symmetric, lower-semibounded quadratic forms $q_{A}$ and $q_{B}$ with form domains $Q(A)$ and $Q(B)$, respectively. We suppose that $q_{A} \leq q_{B}$. This inequality means that $Q(B) \subset Q(A)$ and that, for all $\varphi \in Q(B)$, we have $q_{A}(\varphi) \leq q_{B}(\varphi)$. Under these conditions, Kato proved in [8, Theorem 2.21, chapter VI] the following relationship between the resolvents of the two operators $A$ and $B$. For all $a>-\inf \sigma(A)$, we have

$$
(B+a)^{-1} \leq(A+a)^{-1} .
$$

This resolvent inequality may be used to derive several interesting relations between the traces of functions of $A$ and $B$ under some additional assumptions. We will prove that if $f \geq 0$ and $g$ is a member of a class of functions $\mathcal{L}$ described

Copyright 2016 by the Tusi Mathematical Research Group.

Received Aug. 3, 2015; Accepted Dec. 1, 2015.

${ }^{*}$ Corresponding author.

2010 Mathematics Subject Classification. Primary 47B10; Secondary 47A60, 47B80.

Keywords. operator trace inequalities, operator monotone functions, Loewner's Theorem, random Schrodinger operators, Wegner estimate. 


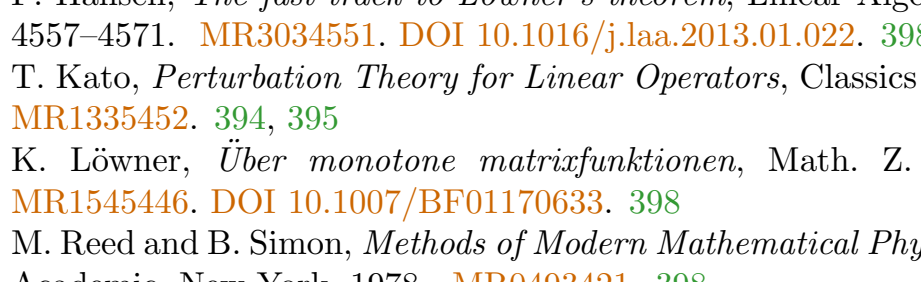

\title{
SOME TRACE MONOTONICITY PROPERTIES AND APPLICATIONS
}

\author{
JEAN-MICHEL COMBES ${ }^{1}$ and PETER D. HISLOP ${ }^{2 *}$ \\ Communicated by I. M. Spitkovsky
}

\begin{abstract}
We present some results on the monotonicity of some traces involving functions of self-adjoint operators with respect to the natural ordering of their associated quadratic forms. The relation between these results and Löwner's Theorem is discussed. We also apply these results to complete a proof of the Wegner estimate for continuum models of random Schrödinger operators as given in a 1994 paper by Combes and Hislop.
\end{abstract}

\section{Statement of the Problem And Result}

We consider two lower-semibounded self-adjoint operators $A$ and $B$ associated with closed symmetric, lower-semibounded quadratic forms $q_{A}$ and $q_{B}$ with form domains $Q(A)$ and $Q(B)$, respectively. We suppose that $q_{A} \leq q_{B}$. This inequality means that $Q(B) \subset Q(A)$ and that, for all $\varphi \in Q(B)$, we have $q_{A}(\varphi) \leq q_{B}(\varphi)$. Under these conditions, Kato proved in [8, Theorem 2.21, chapter VI] the following relationship between the resolvents of the two operators $A$ and $B$. For all $a>-\inf \sigma(A)$, we have

$$
(B+a)^{-1} \leq(A+a)^{-1} .
$$

This resolvent inequality may be used to derive several interesting relations between the traces of functions of $A$ and $B$ under some additional assumptions. We will prove that if $f \geq 0$ and $g$ is a member of a class of functions $\mathcal{L}$ described

Copyright 2016 by the Tusi Mathematical Research Group.

Received Aug. 3, 2015; Accepted Dec. 1, 2015.

${ }^{*}$ Corresponding author.

2010 Mathematics Subject Classification. Primary 47B10; Secondary 47A60, 47B80.

Keywords. operator trace inequalities, operator monotone functions, Loewner's Theorem, random Schrodinger operators, Wegner estimate. 